\title{
Surface-Wave Resonance Effect in a Reactive Cylindrical Structure Excited by an Axial Line Source ${ }^{1}$
}

\author{
A. L. Cullen ${ }^{2}$
}

(July 27, 1959)

\begin{abstract}
It is shown that a purely reactive cylinder excited by a neighboring line source can, under suitable conditions, give rise to a radiation pattern closely approximating the function $\cos n \theta$.

In a numerical example, a cylinder of three transverse electro-magnetic (T.E.M.) wavelengths circumference has a surface reactance chosen to emphasize the term $\cos 6 \theta$ in the Fourier series of the resultant radiation pattern. It is shown that only 1.1 percent of the total power delivered to the line source is radiated in unwanted modes.

It is also shown that the position of the line source does not affect this result to first order provided that $k(b-a) \ll 1$, where $b-a$ is the distance of the line source from the cylindrical surface.
\end{abstract}

\section{Introduction}

In a recent paper the natural (exponentially decaying) oscillations of a highly reactive cylinder supporting a circulating surface wave were discussed [1]. ${ }^{3}$ It was shown that for cylinders of a few wavelengths circumference, Q-factors of the order of several hundred are possible, if the circumferential phase velocity of the surface waves is appreciably less than the velocity of light.

The purpose of the present paper is to examine the corresponding forced oscillations, taking a line source as the means of excitation.

The problem of a line source in the vicinity of a cylinder with a reactive surface impedance has already been solved $[2,3]$ by Wait and Conda, but the reactance values considered in their numerical studies were not such as to exhibit strong resonance effects. Also, they considered a magnetic current line source, whereas for the surface waves discussed in [1] an electric current line source is appropriate. This is a trivial matter, however, since the principle of duality can be employed to pass from one case to the other.

\section{Formulation of the Problem}

The practical interest in strong resonance phenomena lies in the possibility that a radiation pattern of the form $a_{n} \cos n \theta$ might be set up very simply by employing the combination of a line source and a reactive cylindrical surface. Inspection of the general formula shows at once that terms of the form $a_{m} \cos m \theta$ will always be present, but when the surface reactance is chosen to establish resonance with the reactive part of the field impedance of, say, the $n$th mode, we might expect to find $a_{n} \gg a_{m}, m \neq n$.

If we adopt the mean-square-deviation from $a_{n} \cos n \theta$ as the measure of unwanted radiation, it is obvious that we must maximize the following expression:

$$
\eta_{n}=\frac{\frac{1}{2}\left|a_{n}\right|^{2}}{\left|a_{o}\right|^{2}+\frac{1}{2} \sum_{m=1}^{\infty}\left|a_{m}\right|^{2}} \quad(n \neq 0) .
$$

\footnotetext{
1 The research reported in this paper was sponsored by the U.S. Air Force Cambridge Research Center under contract CsO and A $58-40$.

2 Guest researcher from the Department of Electrical Engineering, University of Sheffield, Sheffield, England.

${ }^{3}$ Figures in brackets indicate the literature references at the end of this paper.
} 
The quantity $\eta_{n}$ may be called the "excitation efficiency" or "pattern purity" for the $n$th mode.

The excitation efficiency defined in this way has a very close affinity to the launching efficiency for surface waves of a line source over a plane reactive surface [4]. In this case, the launching efficiency of the surface wave depends markedly on the height $h$ of the source above the reactive surface, the principal effect being a great reduction in radiated power for a certain value of $h$.

It seems reasonable to expect that a similar effect might occur in the cylindrical case if we take $\frac{1}{2}\left|a_{n}^{2}\right|$ as analogous to the surface-wave power and

$$
\left|a_{o}^{2}\right|+\frac{1}{2} \sum_{m=1}^{n-1}\left|a_{m}^{2}\right|+\frac{1}{2} \sum_{n+1}^{\infty}\left|a_{m}^{2}\right|
$$

as analogous to the radiated power in the plane surface wave excitation problem. With this analogy in mind, one might look for an optimum position of the line source which would give the closest approximation, in the least-mean-squares sense, to a $\cos n \theta$ radiation pattern.

\section{Current Sheet Excitation}

Although the necessary formulas have already been given by Wait and Conda, we shall sketch a derivation for completeness. The opportunity will be taken to adapt the point of view employed in [4] to the cylindrical case. That is to say, the line source is synthesized from cylindrical current sheets rather than from point dipoles as in Wait and Conda's work. Electric current sources will be considered rather than magnetic current sources for the reason mentioned earlier.

Referring to figure 1, a sinusoidally distributed cylindrical current sheet of radius $b$ coaxial with the cylinder of radius $a,(a \leqq b)$ is first considered. The current is assumed to flow parallel to the axis, and the current density is written

$$
K_{z}=K_{n} \cos n \theta
$$

where $n$ must clearly be an integer if $K_{z}$ is to be a single-valued function of $\theta$.

The following equations for the fields can then be written down $a \leqq r \leqq b$

$$
\begin{aligned}
& E_{z}^{\prime}=\left[A J_{n}(k r)+B H_{n}(k r)\right] \cos n \theta \\
& H_{\theta}^{\prime}=\frac{1}{j \zeta}\left[A J_{n}^{\prime}(k r)+B H_{n}^{\prime}(k r)\right] \cos n \theta
\end{aligned}
$$

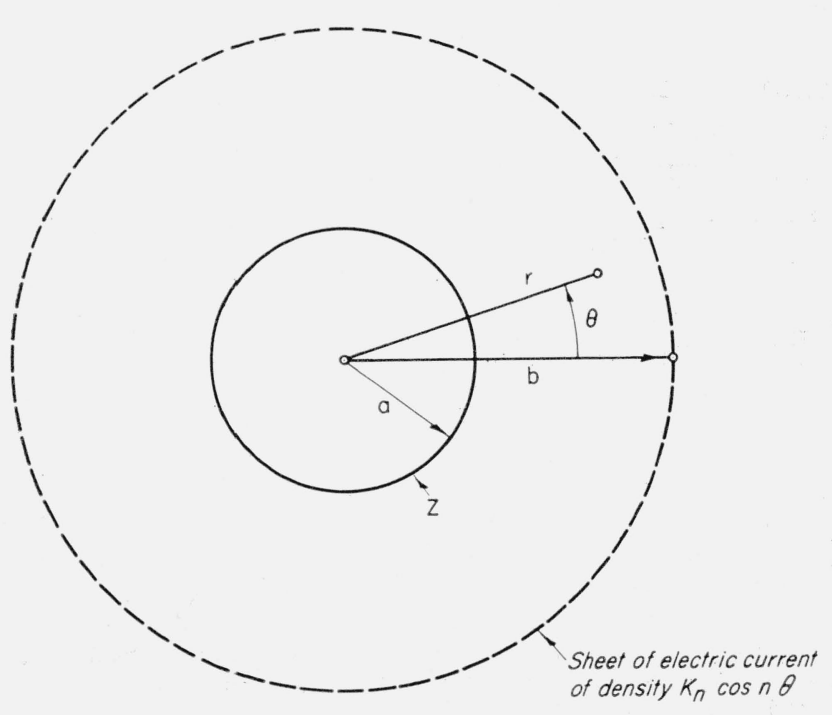

Figure 1. Surface-current sheet excitation for cylinder having finite surface impedance. 


$$
\begin{aligned}
E_{z} & =C H_{n}(k r) \cos n \theta \\
H_{\theta} & =\frac{C}{j \zeta} H_{n}^{\prime}(k r) \cos n \theta
\end{aligned}
$$

In the above equations $H_{n}^{(2)}(k r)$ has been written as $H_{n}(k r)$ to simplify the notation. No confusion should result since the function $H_{n}^{(1)}(k r)$ is not employed in this paper. The quantity $\zeta$ is the intrinsic impedance of free space $\left(\mu_{0} / \epsilon_{0}\right)^{\frac{1}{2}}$.

The constants $A, B$, and $C$ are to be evaluated from the conditions

$$
\text { (i) } E_{z}^{\prime}=Z H_{\theta}^{\prime} \text { at } r=a
$$

where $Z$ is the surface impedance looking into the cylinder.

$$
\begin{aligned}
& \text { (ii) } E_{z}^{\prime}=E_{z} \text { at } r=b \\
& \text { (iii) } H_{\theta}^{\prime}-H_{\theta}=K_{z} \text { at } r=b \text {. }
\end{aligned}
$$

After straightforward algebra, making use of the Wronskian to simplify the term $J_{n} H_{n}^{\prime}-H_{n} J_{n}^{\prime}$ which arises, we arrive at the following expression for $E_{z}$ for $r>b$,

$$
\begin{gathered}
E_{z}=\frac{\pi b}{2} \omega \mu_{0} K_{n}\left[J_{n}(k b)+\alpha_{n} H_{n}(k b)\right] H_{n}(k r) \cos n \theta \\
\alpha_{n}=-\frac{j \zeta J_{n}(k a)-Z J_{n}^{\prime}(k a)}{j \zeta H_{n}(k a)-Z H_{n}^{\prime}(k a)} .
\end{gathered}
$$

Before proceeding to the next step of synthesizing the line source from current sheet sources of the form described by eq (1), we shall examine (7) which plays a role roughly similar to the reflection coefficient $\left(u+k_{1}\right) /\left(u-k_{1}\right)$ of the plane surface wave excitation case [4]. However, the infinite reflection coefficient found for plane surface waves is not to be expected with circulating cylindrical surface waves, for these waves are inherently leaky, and so damping is present even when the impedance $Z$ is purely reactive. In fact we shall show that $\left|\alpha_{n}\right|$ cannot exceed unity.

$\alpha_{n}$ will first be written in terms of $J_{n}$ and $Y_{n}$, putting $Z=j X$.

$$
\alpha_{n}=-\frac{\left[J_{n}(k a)-\frac{X}{\zeta} J_{n}^{\prime}(k a)\right]}{\left[J_{n}(k a)-\frac{X}{\zeta} J_{n}^{\prime}(k a)\right]-\mathrm{j}\left[Y_{n}(k a)-\frac{X}{\zeta} Y_{n}^{\prime}(k a)\right]}
$$

For $n>k a$ pronounced resonance effects occur. $\quad Y_{n}$ and $Y_{n}^{\prime}$ are in general considerably larger than $J_{n}$ and $J_{n}^{\prime}$ so that $\alpha_{n}$ tends to be small unless $Y_{n}(k a)-(X / \zeta) Y_{n}^{\prime}(k a)$ is zero.

For $n<k a$, however, the resonance effect is very weak.

The condition for resonance can be written

When (9) is satisfied

$$
\frac{X}{\zeta}=\frac{Y_{n}(k a)}{Y_{n}^{\prime}(k a)}
$$

$$
\alpha_{n}=-1
$$

If (9) is not satisfied it follows from eq (8) that

$$
\left|\alpha_{n}\right|<1 \text {. }
$$


Thus, we have proved the statement made earlier that the quantity $\alpha_{n}$ is never infinite, and in fact cannot exceed unity.

A closer analogy with reflection coefficient is the complex ratio of outgoing to incoming cylindrical waves in the region $a<r<b$. This is equal to $1+2 \alpha_{m}$, and the reflection coefficient in this sense can never exceed unity in magnitude.

As a numerical example of the way the coefficients $\alpha_{n}$ depend on $n,\left|\alpha_{n}\right|^{2}$ has been calculated as a function of $n$ for the case in which $X / \zeta$ is chosen to satisfy the resonance condition, eq (9), for $n=6$, taking $k a=3$. The appropriate value of $X / \zeta$ is -0.605 . The results are given in table 1 .

TABLE 1

\begin{tabular}{c|c}
\hline$n$ & $\left|\alpha_{n}{ }^{2}\right|$ \\
\cline { 1 - 1 } 0 & 0.869 \\
1 & .054 \\
2 & .970 \\
3 & .641 \\
4 & .151 \\
5 & .018 \\
6 & 1.000 \\
7 & $1.4 \times 10^{-6}$ \\
\hline
\end{tabular}

The very rapid falling-off of $\left|\alpha_{n}\right|^{2}$ with $n$ for $n$ larger than the resonant value is indicated by the last line of this table.

The sharpness of the resonance of $\left|\alpha_{6}\right|^{2}$ as $X / \zeta$ is varied is shown in figure 2 .

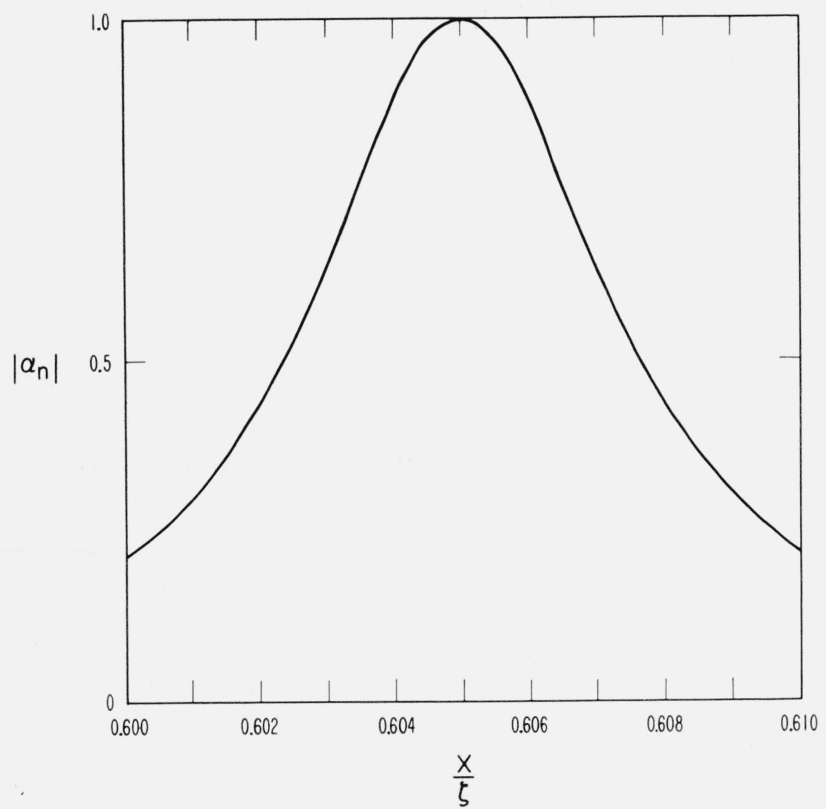

FIgURE 2. Variation of $\left|\alpha_{n}\right|$ with $\mathrm{x} / \zeta$, showing the effect of surface-wave resonance.

If we define a $Q$-factor for the variation of $\left|\alpha_{n}\right|^{2}$ with $X$ through the equation

$$
\left|a_{n}\right|^{2} \cong \frac{1}{1+\left(Q_{x} \frac{\delta X}{X_{0}}\right)^{2}}
$$

the approximate $Q$-factor deduced from figure 2 is $Q_{x}=230$.

This is considerably lower than the $Q$-factor deduced in [1], but the conditions are somewhat different. 
The difference lies in the way the reactances involved depend on the frequency. In [1] it is assumed that the capacitive surface reactance $X$ is inversely proportional to the frequency, while the inductively reactive part of the field impedance increases with frequency, in accordance with field theory, more rapidly than in direct proportion to the frequency.

The Q-factor $Q_{x}$ defined by (12) would only agree with the Q-factor of [1] if the reactive part of the field impedance varied in direct proportion to the frequency.

Hence, the Q-factor of [1] will necessarily exceed the rather artificial $Q_{x}$ introduced here.

\section{Line Source Excitation}

Suppose we have a linear current filament $I$ at $r=b, \theta=0$. This can be represented as a Fourier series thus

$$
\frac{I \delta(\theta)}{b}=\sum_{m=0}^{\infty} K_{m} \cos m \theta
$$

The coefficients $K_{m}$ represent the amplitudes of cosinusoidally distributed surface current densities, and are found in the usual way to be

where $\epsilon_{0}=1, \epsilon_{m}=2, m \neq 0$.

$$
K_{m}=\epsilon_{m} \frac{I}{2 \pi b}
$$

The field associated with each $K_{m}$ can be found from eq (6), and in this way we find the formula for current filament excitation

$$
E_{z}=\frac{\omega \mu_{0} I}{4} \sum_{m=0}^{\infty} \epsilon_{m}\left[J_{m}(k b)+\alpha_{m} H_{m}(k b)\right] H_{m}(k r) \cos m \theta .
$$

This formula makes possible far-field calculations, and for this purpose $H_{m}(k r)$ can be replaced by its asymptotic value.

$$
H_{m}(k r) \cong\left(\frac{2}{\pi k r}\right)^{\frac{1}{2}} \exp \left[-j\left(k r-\frac{1}{2} m \pi-\frac{1}{4} \pi\right)\right]
$$

Equation (15) is the dual of the formula derived by Wait [3] and is identical apart from minor differences of notation if the following transformations are made: $E_{z} \rightarrow H_{z}, \mu_{0} \rightarrow \epsilon_{0}$, and $I \rightarrow K$ (symbol used to denote magnetic current). It may also be derived from Wait's result [5] for the fields of an electric dipole in the presence of a cylinder with impedance boundary conditions.

Equation (15) enables us to calculate the pattern purity or excitation efficiency according to the definition given in the introduction to this paper. The asymptotic value of $H_{m}(\mathrm{kr})$ given by (16) has the magnitude $(2 / \pi k r)^{1 / 2}$ for all values of $m$, and therefore we may take as our $a_{m}$ 's the quantities $\epsilon_{m}\left[J_{m}(k b)+\alpha_{m} H_{m}(k b)\right]$.

Thus we find

$$
\eta_{n}=\frac{2\left|J_{n}(k b)+\alpha_{n} H_{n}(k b)\right|^{2}}{\sum_{m=0}^{\infty} \epsilon_{m}\left|J_{m}(k b)+\alpha_{m} H_{m}(k b)\right|^{2}} \quad(n \neq 0) .
$$

The special case $b=a$ is worthy of attention. In this case the term in square brackets in eq (15) can be simplified by making use of the Wronskian again, to read

$$
\frac{2 j}{\pi k a}\left[\frac{\frac{X}{\zeta}}{H_{n}(k a)-\frac{X}{\zeta} H_{n}^{\prime}(k a)}\right]
$$


and eq (15) then becomes

$$
E_{z}=\frac{j \zeta I}{2 \pi a} \sum_{m=0}^{\infty} \epsilon_{m}\left[\frac{\frac{X}{\zeta}}{H_{m}(k a)-\frac{X}{\zeta} H_{m}^{\prime}(k a)}\right] H_{m}(k r) \cos m \theta .
$$

The eq (17) for excitation efficiency can be specialised for $b=a$ to read

$$
\eta_{n}=\frac{2\left|\frac{1}{H_{n}(k a)-\frac{X}{\zeta} H_{n}^{\prime}(k a)}\right|^{2}}{\sum_{m=0}^{\infty} \epsilon_{m}\left|\frac{1}{H_{m}(k a)-\frac{X}{\zeta} H_{m}^{\prime}\left(k_{a}\right)}\right|^{2}} .
$$

Let us now consider (17) for the case $k(b-a)<<1$.

We can write

$$
J_{m}(k b)+\alpha_{m} H_{m}(k b) \cong\left\{J_{m}(k a)+\alpha_{m} H_{m}(k a)\right\}+\left\{J_{m}^{\prime}(k a)+\alpha_{m} H_{m}^{\prime}(k a)\right\} k(b-a)
$$

if terms of order $k^{2}(b-a)^{2}$ are neglected. We can use (18) to replace the first term on the right-hand side of this equation, and by a similar simplification of the second term we find

$$
J_{m}(k b)+\alpha_{m} H_{m}(k b)=\frac{2 j}{\pi k a}\left[\frac{\frac{X}{\zeta}}{H_{n}(k a)-\frac{X}{\zeta} H_{n}^{\prime}(k a)}\right] \times\left\{1+\frac{\zeta}{X} k(b-a)\right\} .
$$

The last factor in (21) is independent of $m$, and so to a first approximation we find that all noodes are multiplied by the same factor. Thus, although a small finite value of $k(b-a)$ will alter the total power supplied by the source for a given current, and so will alter the radiation resistance of the line source, it will not affect the distribution of power among the various modes, and so will not change the excitation efficiency.

Thus it follows that eq (20) is valid for $b \neq a$, provided that $k(b-a)<<1$.

\section{Example of Excitation Efficiency Calculation}

We now consider the excitation efficiency of the mode $n=6$ by a line source near the cylinder, i.e., $k(b-a)<<1$, for the case $k a=3$. The surface reactance is again chosen to establish resonance for the mode $n=6$. Remembering that excitation efficiency, or pattern purity, is defined as power in the wanted mode divided by the total radiated power, i.e., in symbols

$$
\eta_{n}=\frac{P_{n}}{\sum_{m=1}^{\infty} P_{m}}
$$

it is instructive to tabulate the $P_{m}$ 's in order to find how the unwanted radiation is distributed amongst the various modes. This information is given in table 2 .

\begin{tabular}{c|r}
\multicolumn{2}{c}{ TARLE 2 } \\
\hline$m$ & \multicolumn{1}{c}{$P_{m}$} \\
\hline & \\
\hline 0 & 4.02 \\
1 & 8.00 \\
2 & 7.85 \\
3 & 7.36 \\
4 & 6.74 \\
5 & 6.78 \\
6 & 3580. \\
7 & 0.08 \\
&
\end{tabular}


It appears that very little power is radiated in modes for which $m>6$, and that for $m<6$, the power is fairly equally divided between the various modes.

Only 1.1 percent of the total power goes into unwanted modes, and the excitation efficiency has the very high value of 98.9 percent.

\section{Conclusions}

The existence of strong resonance phenomena associated with surface waves circulating round a reactive cylinder excited by a line source has been established theoretically. In a particular case, 98.9 percent of the total input power is radiated in the resonant mode, i.e., the excitation efficiency, or pattern purity is 98.9 percent.

It is shown that to first order in $k(b-a)$, the distance of the line source from the surface of the cylinder does not affect the excitation efficiency, though the radiation resistance of the source will decrease as $k(b-a)$ increases.

This paper was written during a short visit to the Boulder Laboratories of the National Bureau of Standards. The author expresses his gratitude for this most stimulating opportunity, particularly to James R. Wait of these laboratories through whom the visit was arranged.

\section{References}

[1] M. F. Bracey, A. L. Cullen, E. F. F. Gillespie, and J. A. Staniforth, Surface wave research in Sheffield, Proc. of URSI Symposium on Electromag. Wave Theory (1959).

[2] J. R. Wait and Alyce M. Conda, Radiation from slots on dielectric clad and corrugated cylinders, J. Research NBS, 59, 307 (1957) RP2802.

[3] J. R. Wait, Electromagnetic radiation from cylindrical structures, p. 135 (Pergamon Press, New York, N.Y., 1959).

[4] A. L. Cullen, The excitation of plane surface waves, Proc. Inst. Elec. Engrs. Part IV, 101, 225 (1954).

[5] J. R. Wait, Radiation from an electric dipole in the presence of a corrugated cylinder, Appl. Sci. Research, Sec. B, 6, 117 (1956).

Boulder, Colo.

(Paper 64D1-32) 